\title{
LIMIT BEHAVIOR OF LOCALLY CONSISTENT CONSTRAINT SATISFACTION PROBLEMS
}

\author{
MANUEL BODIRSKY* AND DANIEL KRÁL’†
}

\begin{abstract}
An instance of a constraint satisfaction problem (CSP) is variable $k$-consistent if any subinstance with at most $k$ variables has a solution. For a fixed constraint language $\mathcal{L}, \rho_{k}(\mathcal{L})$ is the largest ratio such that any variable $k$-consistent instance has a solution that satisfies at least a fraction of $\rho_{k}(\mathcal{L})$ of the constraints. We provide an expression for the limit $\rho(\mathcal{L}):=\lim _{k \rightarrow \infty} \rho_{k}(\mathcal{L})$, and show that this limit coincides with the corresponding limit for constraint $k$-consistent instances, i.e., instances where all subinstances with at most $k$ constraints have a solution. We also design an algorithm running in time polynomial in the size of input and $1 / \varepsilon$ that for an input instance and a given $\varepsilon$ either computes a solution that satisfies at least a fraction of $\rho(\mathcal{L})-\varepsilon$ constraints or finds a set of inconsistent constraints whose size only depends on $\epsilon$. Most of our results apply both to weighted and to unweighted instances of the constraint satisfaction problem.
\end{abstract}

Key words. Constaint satisfaction problem (CSP), local consistency

AMS subject classifications. 68R05, 05D40

1. Introduction. Constraint satisfaction problems (CSPs) form an important computational model for problems arising in many areas of computer science. This is witnessed by an enormous interest in the computational complexity of the problem and its variants $[2,3,5-7,9-11,13,14,16,17,21,30]$. However, sometimes not all the constraints of an input instance need to be satisfied, but it suffices to satisfy a large fraction of them. A natural notion in this context is the notion of local consistency. A CSP instance is constraint $k$-consistent if for any $k$ constraints of the instance, there exists an assignment of the values to the variables such that the $k$ chosen constraints are all satisfied. Similarly, a CSP instance is variable $k$-consistent if any $k$ variables of the instance can be assigned values in such way that no constraint that contains only those $k$ variables is violated by the assignment. In this paper, we focus on the effect of local consistency on the quality of a global optimal solution with respect to the number of constraints that can be simultaneously satisfied. There is an interesting connection between this question and the notion of tree-duality, which has been studied in graph theory [16] and recently in logic [24].

Let us remark that there are several other notions of local consistency that are different from the notion that we analyze, such as the notion of $k$-consistency introduced by Freuder [13], or the notion of relational k-consistency studied by Dechter and van Beek [6]. However, we do not address any of these notions in this paper.

1.1. Previous Results. The notion of local consistency considered in this paper can be traced back to the early 1980's. Lieberherr and Specker $[25,26]$ studied the corresponding problem for $\mathrm{CNF}$ formulas: they require that any $k$ clauses of a given formula can be satisfied and asked what fraction of the clauses can be satisfied. In their papers, they settled the cases $k=1,2,3$ and showed that the corresponding fractions are $1 / 2,(\sqrt{5}-1) / 2$ and $2 / 3$ (the values are now known for $k \geq 4$ ). A

${ }^{*}$ Laboratoire d'Informatique (LIX), CNRS UMR 7161, École Polytechnique, 91128 Palaiseau, France. E-mail: bodirsky@lix.polytechnique.fr.

$\dagger$ Institute for Theoretical Computer Science (ITI), Faculty of Mathematics and Physics, Charles University, Malostranské náměstí 25, 11800 Prague 1, Czech Republic. E-mail: kral@kam.mff.cuni.cz. Institute for Theoretical computer science is supported as project 1M0545 by Czech Ministry of Education. 
simpler proof of their results was later found by Yannakakis [31]. The case $k=4$ was settled in [22]. There is an interesting connection between this problem and Usiskin numbers [29]. Locally consistent CNF formulas are also discussed in Chapter 20 of the monograph [20].

The asymptotic behavior of locally consistent CNF formulas when $k$ approaches infinity was first addressed by Huang et al. [18] and further studied by Trevisan [28]. Trevisan [28] was the first to define the notion of local consistency with constraints being Boolean predicates. For a set $\Pi$ of Boolean constraints, i.e., the domain is not just a two-element set but the Boolean field, let $\rho_{k}(\Pi)$ be the maximum $\rho$ such that a fraction of at least $\rho$ constraints can be satisfied for any $k$-consistent input. In this scenario, it is often assumed that negations of the arguments of the predicates are also allowed in the input. This changes the problem significantly, since the constraint language is "closed" under taking negations of arguments of its predicates; this leads to a simpler analysis without using set structures.

Let us survey the results known for the Boolean case. The $\operatorname{limit}_{\lim } \lim _{k \rightarrow \infty} \rho_{k}(\Pi)$ for a set $\Pi$ of all predicates of arity $\ell$ is equal $2^{1-\ell}[28]$. The ratios $\rho_{k}(\Pi), k \geq 1$, for a set $\Pi$ consisting of a single predicate of arity at most three were determined by Dvoŕák et al. [8]. The asymptotic behavior of $\rho_{k}(\Pi)$ for fixed sets $\Pi$ of predicates was studied in [23], and $\lim _{k \rightarrow \infty} \rho_{k}(\Pi)$ was expressed as the minimum of a certain functional on a convex set of polynomials derived from $\Pi$; this expression and its derivation is simpler than the one presented in this paper for the reason we mentioned in the previous paragraph. Efficient algorithms for locally consistent CSPs with constraints that are Boolean predicates were also designed $[8,23]$.

1.2. Our Results. In the conference version of this paper, we studied CSPs with a single binary constraint type [1] with an arbitrary finite domain. We further develop the methods used there to address the problem in full generality and provide an analysis for all constraint languages. In other words, the constraint language can now contain several types of constraints, which are not necessarily binary, and there is no restriction on the domain as in the previous results which mostly apply to the Boolean domain only.

We now briefly summarize our results. Formal definitions of the mentioned quantities are given in Section 2, and the rigorous statements of the achieved results can be found in the subsequent sections.

Let $\mathcal{L}$ be a fixed finite constraint language. Then $\rho_{v, k}(\mathcal{L})$ denotes the largest value $\xi_{v}$ such that any variable $k$-consistent instance has a solution that satisfies at least a fraction $\xi_{v}$ of the constraints. Let $\rho_{v}(\mathcal{L})$ be the limit of $\rho_{v, k}(\mathcal{L})$ for $k$ tending to infinity. In Section 3, we introduce a parameter $\pi(\mathcal{L})$ of the constraint language $\mathcal{L}$, and later show that $\rho_{v}(\mathcal{L})$ equals $\pi(\mathcal{L})$. Similarly, we introduce $\rho_{c, k}(\mathcal{L})$ as the largest fraction $\xi_{c}$ such that any constraint $k$-consistent instance has a solution that satisfies at least a fraction $\xi_{c}$ of the constraints. We show that the limit of this quantity also equals $\pi(\mathcal{L})$. Unless the language $\mathcal{L}$ contains a unary constraint, the result applies to both weighted and unweighted instances. In case that $\mathcal{L}$ contains a unary constraint, our results apply only to weighted instances.

Next, we develop techniques to find solutions that satisfy many constraints of an instance. In Section 4, we design an efficient algorithm that either constructs a solution of an input instance that satisfies at least a fraction of $\rho_{v}(\mathcal{L})-\varepsilon$ of the constraints or finds an inconsistent set of constraints of size bounded by a function that only depends on $\varepsilon$.

In Section 5, we address the difference between weighted and unweighted instances 
of the CSP. Note that we do not allow repetition of the same constraint in unweighted instances to simulate weights. We show that if the constraint language does not contain a unary constraint, then weighted and unweighted locally consistent problems have the same extremal behavior.

Finally, in Section 6, we use our results to derive the results obtained in the conference version of this paper for constraint languages with a single binary constraint type. Results for such constraint languages are of particular interest, since the constraint satisfaction problem then corresponds to the (directed) graph homomorphism problem. In particular, we prove that $\rho(\mathcal{L})=1$ if and only if $\mathcal{L}$ has tree duality. The concept of tree-duality for (directed) graphs is well-studied in graph theory [16].

2. Notation and Definitions. A (finite) constraint language is a pair $\mathcal{L}=$ $(D, \mathcal{U})$ such that $D$ is a finite domain and $\mathcal{U}=\left\{U_{1}, \ldots, U_{k}\right\}$ is a finite set of relations on $D$, i.e., $U_{i} \subseteq D^{r_{i}}$ for some positive integer $r_{i}$. The value of $r_{i}$ is the arity of the relation $U_{i}$. Relations of $\mathcal{U}$ are called constraint types.

An instance of a constraint satisfaction problem with the constraint language $(D, \mathcal{U})$ is a pair $(X, \mathcal{R})$ such that $X$ is the set of variables and $\mathcal{R}=\left\{R_{1}, \ldots, R_{m}\right\}$ is the set of constraints. Each $R_{i}$ is an ordered $r_{j}$-tuple of the (not necessarily distinct) elements of $X$, and $R_{i}$ is associated with a constraint type $U \in \mathcal{U}$ of arity $r_{j}$. Inspired by terminology in graph theory, we say that an instance is simple if each constraint in $\mathcal{R}$ contains each variable at most once. We say that a mapping $\varphi: X \rightarrow D$ satisfies a constraint $R_{i}=\left(x_{1}, \ldots, x_{r_{j}}\right)$ of constraint type $U_{j}$ if $\left(\varphi\left(x_{1}\right), \ldots, \varphi\left(x_{r_{j}}\right)\right) \in U_{j}$. Mappings $\varphi$ from $X$ to $D$ will be called solutions of an instance; an exact solution is a solution that satisfies all the constraints of the instance.

A weighted instance is an instance such that each constraint $R_{i}$ is assigned a nonnegative weight $w\left(R_{i}\right)$. The total weight $w_{0}$ of an instance is the sum of the weights of all its constraints. The weight $w(\varphi)$ of a solution $\varphi: X \rightarrow D$ is the sum of the weights $w\left(R_{i}\right)$ of the satisfied constraints $R_{i}$, i.e., those constraints $\left(x_{1}, \ldots, x_{r_{j}}\right)$ with $\left(\varphi\left(x_{1}\right), \ldots, \varphi\left(x_{r_{j}}\right)\right) \in U_{j}$. An optimum solution is a solution of maximum weight. Finally, if all the constraints are assigned the same weight, the instance is called uniform; uniform instances correspond to unweighted ones.

An important class of constraint satisfaction problems are those corresponding to graph homomorphism problems. If $G$ and $H$ are graphs, then a homomorphism from $G$ to $H$ is a mapping $\varphi: V(G) \rightarrow V(H)$ where $\varphi(u) \varphi(v) \in E(H)$ for every edge $u v \in E(G)$. The same definition applies both to undirected and directed graphs. The problem of deciding an existence of a homomorphism to a graph $H$ corresponds to the constraint language $(D, \mathcal{U})$ where $D=V(H)$ and $\mathcal{U}$ consists of a single binary constraint type $U_{1}$ such that $(u, v) \in U_{1}$ if and only if $u v \in E(H)$. The instance $(X, \mathcal{R})$ corresponding to an input graph $G$ consists of $|V(G)|$ variables $x_{v}$ corresponding to vertices $v \in V(G)$, and $\mathcal{R}$ contains a binary constraint $\left(x_{u}, x_{v}\right)$ for every edge $u v \in E(G)$. It is easy to see that exact solutions of the constructed instance of CSP are in one-to-one correspondence with homomorphisms from $G$ to $H$.

As in the case of graphs and graph homomorphisms, one may view general constraint satisfaction problems as homomorphism problems for relational structures [11, 21]. Let $(X, \mathcal{R})$ and $\left(X^{\prime}, \mathcal{R}^{\prime}\right)$ be two instances with the same constraint language $\mathcal{L}$. A mapping $\psi: X \rightarrow X^{\prime}$ is a homomorphism from $(X, \mathcal{R})$ to $\left(X^{\prime}, \mathcal{R}\right)$ if for every constraint $\left(x_{1}, \ldots, x_{r}\right)=R_{i} \in \mathcal{R}$ of type $U_{i}$, there is a constraint $R_{i}^{\prime}=\left(\psi\left(x_{1}\right), \ldots, \psi\left(x_{r}\right)\right) \in \mathcal{R}^{\prime}$ of type $U_{i}$. If such a homomorphism exists, we say that $(X, \mathcal{R})$ is homomorphic to $\left(X^{\prime}, \mathcal{R}\right)$. It is not hard to see that if $(X, \mathcal{R})$ is homomorphic to $\left(X^{\prime}, \mathcal{R}^{\prime}\right)$ and $\left(X^{\prime}, \mathcal{R}^{\prime}\right)$ has an exact solution $\varphi^{\prime}$, then $(X, \mathcal{R})$ has also an exact solution - set $\varphi(x)=\varphi^{\prime}(\psi(x))$ 

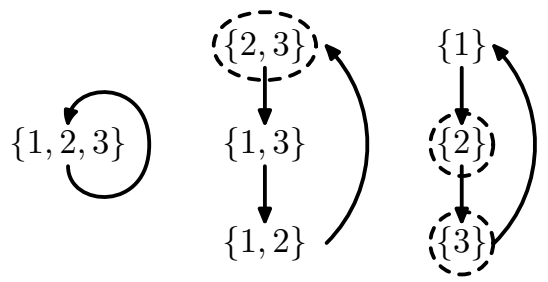

FIG. 2.1. An example of the set structure of a constraint language. The domain of the language is $D=\{1,2,3\}$, and the language contains two relations $R_{1}$ and $R_{2}$, where $R_{1}$ is the unary relation $\{x \mid x \neq 1\}$, and $R_{2}$ is the binary relation $\{[x, y] \mid x=y+1(\bmod 3)\}$. The relation $R_{1}$ is depicted by dashed circles and $R_{2}$ by arrows in the figure.

for every $x \in X$.

In this paper, we address locally consistent constraint satisfaction problems. An instance $(X, \mathcal{R})$ of a constraint satisfaction problem is variable $k$-consistent if $\left(X^{\prime}, \mathcal{R}^{\prime}\right)$ has an exact solution for every set $X^{\prime}$ of at most $k$ elements of $X$ and $\mathcal{R}^{\prime}$ containing all the constraints of $\mathcal{R}$ that include only the variables of $X^{\prime}$. Similarly, an instance is constraint $k$-consistent if $\left(X, \mathcal{R}^{\prime}\right)$ has an exact solution for every subset $\mathcal{R}^{\prime} \subseteq \mathcal{R}$ with at most $k$ elements.

Let $\mathcal{L}$ be a constraint language. We define $\rho_{v, k}^{w}(\mathcal{L})$ to be the largest ratio $\alpha$ such that every variable $k$-consistent instance with the language $\mathcal{L}$ with total weight $w_{0}$ has a solution of weight at least $\alpha w_{0}$. Similarly, $\rho_{c, k}^{w}(\mathcal{L})$ is the largest such ratio for constraint $k$-consistent instances. Analogously, we define ratios $\rho_{v, k}(\mathcal{L})$ and $\rho_{c, k}(\mathcal{L})$ for uniform instances with the language $\mathcal{L}$ (these ratios correspond to the unweighted case). Finally, we define the notation for the limit values:

$$
\rho_{v}^{w}(\mathcal{L})=\lim _{k \rightarrow \infty} \rho_{v, k}^{w}(\mathcal{L}) .
$$

Similarly, we use $\rho_{c}^{w}(\mathcal{L}), \rho_{v}(\mathcal{L})$ and $\rho_{c}(\mathcal{L})$.

An important notion in our considerations is the concept of the set structure for a constraint language $[5,11]$. It is the counterpart to set graphs studied in the area of graph homomorphisms [16].

Definition 2.1. The set structure $2^{\mathcal{L}}$ of a constraint language $\mathcal{L}=(D, \mathcal{U})$ is an instance of $\mathcal{L}$ with $2^{|D|}-1$ variables $x_{A}$, each corresponding to a non-empty subset $A \subseteq D$. For every constraint type $U_{i} \in \mathcal{U}$ with arity $r_{i}$, the set structure $2^{\mathcal{L}}$ contains an $r_{i}$-tuple $\left(x_{A_{1}}, \ldots, x_{A_{r_{i}}}\right)$ as a constraint of type $U_{i}$ if and only if the following holds: for every $j, 1 \leq j \leq r_{i}$, and every $y_{j} \in A_{j}$, there exist $y_{1} \in A_{1}, \ldots, y_{j-1} \in A_{j-1}, y_{j+1} \in$ $A_{j+1}, \ldots, y_{r_{i}} \in A_{r_{i}}$ such that $\left(y_{1}, \ldots, y_{r_{i}}\right) \in U_{i}$.

The set structure $2^{\mathcal{L}}$ is not simple in general. An example of the set structure of a constraint language can be found in Figure 2.1. The importance of the concept of the set structure arises from the fact that if the set structure $2^{\mathcal{L}}$ of a language $\mathcal{L}$ has an exact solution, then the CSP for $\mathcal{L}$ can be solved in polynomial time by the arc-consistency procedure [16]. It is known that $2^{\mathcal{L}}$ has an exact solution if and only if $\mathcal{L}$ has tree duality $[11,17]$.

In our proofs, we often use probabilistic arguments involving Markov's inequality and Chernoff's inequality. We recall these two well-known results from probability theory for the reader's convenience, and refer to [15] for further details.

Proposition 2.2. Let $X$ be a non-negative random variable with expected value 
E. The following holds for every $\alpha \geq 1$ :

$$
\operatorname{Prob}(X \geq \alpha) \leq \frac{E}{\alpha} .
$$

Proposition 2.3. Let $X$ be a random variable for the sum of $N$ zero-one independent random variables each of which is equal to 1 with probability $p$. Then the following holds for every $0<\delta \leq 1$ :

$$
\operatorname{Prob}(X \geq(1+\delta) p N) \leq e^{-\frac{\delta^{2} p N}{3}} \quad \text { and } \quad \operatorname{Prob}(X \leq(1-\delta) p N) \leq e^{-\frac{\delta^{2} p N}{2}} .
$$

3. Upper Bounds on $\rho$. In this section, we prove upper bounds on the limits $\rho_{v}^{w}(\mathcal{L}), \rho_{c}^{w}(\mathcal{L}), \rho_{v}(\mathcal{L})$ and $\rho_{c}(\mathcal{L})$. (And in the next section we will prove the matching lower bounds.) To achieve our goal, we have to construct a locally consistent instance with constraint language $\mathcal{L}$. One way of proving that a given instance is locally consistent is to show that it is homomorphic to $2^{\mathcal{L}}$ and that it does not have short cycles. A cycle of an instance $(X, \mathcal{R})$ is a cyclic sequence of constraints $R_{1}, \ldots, R_{k} \in$ $\mathcal{R}, k \geq 1$, with arity at least two such that any two consecutive constraints share at least one variable. In case that $k=1$ we additionally require that at least two variables in $R_{1}$ coincide. The number $k$ of constraints is called the length of the cycle. Cycles of length 1 are also called loops. A cycle is minimal if no proper subset of its constraints forms a cycle. Note that no minimal cycle can contain a unary constraint. A CSP instance $(X, \mathcal{R})$ is acyclic if it does not contain a cycle. In particular, acyclic instances do not contain loops.

An instance is not homomorphic to the set structure if and only if the so-called arcconsistency procedure detects an unsatisfiable set of constraints in the instance $[5,12]$. Together with the well-known fact that the arc-consistency procedure solves acyclic instances of the constraint satisfaction problem (see $[7,14]$ for stronger results on bounded tree-width instances), this implies the following lemma.

Lemma 3.1. Let $(X, \mathcal{R})$ be an acyclic CSP instance with constraint language $\mathcal{L}$. If $(X, \mathcal{R})$ is homomorphic to $2^{\mathcal{L}}$, then $(X, \mathcal{R})$ has an exact solution.

Next, we show how Lemma 3.1 can be applied to show that an instance with no short cycles is locally consistent:

LEMMA 3.2. Let $(X, \mathcal{R})$ be an instance of $\operatorname{CSP}(\mathcal{L})$. If $(X, \mathcal{R})$ is homomorphic to $2^{\mathcal{L}}$ and $(X, \mathcal{R})$ does not have a cycle of length at most $k$, then $(X, \mathcal{R})$ is both variable and constraint $k$-consistent.

Proof. First, we show that $(X, \mathcal{R})$ is constraint $k$-consistent. Let $R_{1}, \ldots, R_{k} \in \mathcal{R}$ be $k$ constraints from the instance. Since $(X, \mathcal{R})$ does not have a cycle of length at most $k$, the instance $\left(X, \mathcal{R}^{\prime}\right)$ with $\mathcal{R}^{\prime}=\left\{R_{1}, \ldots, R_{k}\right\}$ is acyclic. It is easy to observe that $\left(X, \mathcal{R}^{\prime}\right)$ is homomorphic to $2^{\mathcal{L}}$, since $(X, \mathcal{R})$ is homomorphic to $2^{\mathcal{L}}$, and since $\mathcal{R}^{\prime} \subseteq \mathcal{R}$. By Lemma 3.1, there is an exact solution of $\left(X, \mathcal{R}^{\prime}\right)$.

Next, we show that $(X, \mathcal{R})$ is variable $k$-consistent. Let $X^{\prime} \subseteq X$ be any subset of $X$ with $k$ variables, and let $\mathcal{R}^{\prime} \subseteq \mathcal{R}$ be the set of all the constraints of $(X, \mathcal{R})$ that contain only the variables of $X$. If $\left(X^{\prime}, \mathcal{R}^{\prime}\right)$ contains a cycle, it also contains a minimal cycle. The length of a minimal cycle cannot exceed $\left|X^{\prime}\right|=k$. Since $(X, \mathcal{R})$ does not have a cycle of length at most $k,\left(X^{\prime}, \mathcal{R}^{\prime}\right)$ is acyclic. Again, we know that $\left(X, \mathcal{R}^{\prime}\right)$ is homomorphic to $2^{\mathcal{L}}$ and it has an exact solution by Lemma 3.1.

Our bounds on $\rho_{v}^{w}(\mathcal{L}), \rho_{c}^{w}(\mathcal{L}), \rho_{v}(\mathcal{L})$, and $\rho_{c}(\mathcal{L})$ are given in terms of the following parameter $\pi(\mathcal{L})$ of a constraint language $\mathcal{L}=(D, \mathcal{U})$. 
Definition 3.3. Let $\mathcal{L}$ be a constraint language and $2^{\mathcal{L}}=(X, \mathcal{R})$ its set structure. Let $\mathcal{W}$ be the set of all non-negative weight functions $w: \mathcal{R} \rightarrow[0, \infty)$ with total weight equal to one and $\mathcal{P}$ the set of functions the maximum is taken over all functions $p: X \times D \rightarrow[0,1]$ that satisfy

$$
\sum_{d \in D} p(x, d)=1
$$

for every variable $x \in X$ of the set structure $2^{\mathcal{L}}$. The following quantity is denoted by $\pi(\mathcal{L})$.

$$
\min _{w \in \mathcal{W}} \max _{p \in \mathcal{P}} \sum_{R_{i}=\left(x_{1}, \ldots, x_{r_{i}}\right) \in \mathcal{R}} w\left(R_{i}\right) \cdot \sum_{\left(d_{1}, \ldots, d_{r_{i}}\right) \in U\left(R_{i}\right)} \prod_{k=1}^{r_{i}} p\left(x_{k}, d_{k}\right)
$$

where $U\left(R_{i}\right)$ is the constraint type of the constraint $R_{i}$.

Note that the inner quantity of the expression (3.1) equals the expected weight of a solution $\varphi: X \rightarrow D$ of $2^{\mathcal{L}}=(X, \mathcal{R})$ that assigns a value $d \in D$ to the variable $x \in X$ with probability $p(x, d)$. Hence, $\pi(\mathcal{L})$ is equal to the maximum expected weight of a "random" solution $\varphi$ for the "worst" constraint weights $w$.

It is easy to verify that both the minimum and the maximum in (3.1) are attained: for fixed $w$, the sum over all constraints in the set structure in (3.1) is a continuous function in $p$ which is defined on a compact set. Hence, the maximum is attained. This maximum is again a continuous function in $w$, and since $\mathcal{W}$ is also a compact set, the minimum is attained as well.

Also observe that $2^{\mathcal{L}}=(X, \mathcal{R})$ for a constraint language $\mathcal{L}=(D, \mathcal{U})$ has an exact solution if and only if $\pi(\mathcal{L})=1$. Indeed, if $2^{\mathcal{L}}$ has an exact solution $\varphi: X \rightarrow D$, then the maximum in (3.1) is attained for

$$
p(x, d)= \begin{cases}1 & \text { if } \varphi(x)=d, \text { and } \\ 0 & \text { otherwise }\end{cases}
$$

since the product $\prod_{k=1}^{r_{i}} p\left(x_{k}, d_{k}\right)$ in the definition equals one for all the constraints $R_{i} \in \mathcal{R}$, and thus the inner part of $(3.1)$ also equals $w_{0}=1$. Hence, $\pi(\mathcal{L})=1$. On the other hand, if $\pi(\mathcal{L})=1$, then consider the weight function $w$ that assigns $1 / m$ to each constraint of $\mathcal{R}$ where $m=|\mathcal{R}|$. Since $\pi(\mathcal{L})=1$, there exists a function $p$ in $(3.1)$ such that the expected weight of a solution $\varphi: X \rightarrow D$ of $2^{\mathcal{L}}$ that assigns a variable $x \in X$ a value $d \in D$ with probability $p(x, d)$ is equal to $w_{0}=1$. It follows that $2^{\mathcal{L}}$ has an exact solution.

We are now ready to state the main theorem of this section.

TheOrem 3.4. Let $\mathcal{L}=(D, \mathcal{U})$ be a constraint language. The following holds:

$$
\rho_{v}^{w}(\mathcal{L}) \leq \pi(\mathcal{L}) \text { and } \rho_{c}^{w}(\mathcal{L}) \leq \pi(\mathcal{L})
$$

If $\mathcal{L}$ does not contain any unary constraint type, we also have

$$
\rho_{v}(\mathcal{L}) \leq \pi(\mathcal{L}) \text { and } \rho_{c}(\mathcal{L}) \leq \pi(\mathcal{L})
$$

Proof. For a fixed integer $k \geq 1$ and a positive real $0<\varepsilon<1 / 10$, we construct a simple weighted instance $(X, \mathcal{R})$ with total weight $w_{0}$ that is both variable and constraint $k$-consistent, all the non-unary constraints of $\mathcal{R}$ have weight one, and every 
solution $\varphi$ has weight at most $(\pi(\mathcal{L})+\varepsilon) w_{0}$. The existence of such instances yields all the statements of the theorem.

The existence of the sought instance is established in several steps. We first describe a random procedure of constructing an instance $(X, \mathcal{R})$ of $\mathcal{L}$. The construction will almost surely yield an instance which is neither variable nor constraint $k$-consistent. However, we show that $(X, \mathcal{R})$ can be pruned to be locally consistent with removing only a small fraction of the constraints. We then show that $(X, \mathcal{R})$ contains constraints with large weight on average but does not have a solution with large weight. Most of our arguments are based on estimating large deviations from the expected value using Markov's and Chernoff's inequalities.

- Construction. Let $N$ be a sufficiently large integer that we fix later. Let further $\left(X_{0}, \mathcal{R}_{0}\right)$ be the set structure $2^{\mathcal{L}}$, let $w_{\mathcal{L}}$ be the weight function that minimizes the value of (3.1). Recall that the total weight $w_{\mathcal{L}, 0}$ of the constraints of $2^{\mathcal{L}}$ is equal to 1 . The variable set $X_{0}$ of $2^{\mathcal{L}}$ contains $2^{|D|}-1$ variables $x_{A}$, each corresponding to a non-empty subset $A \subseteq D$ (see Definition 3.3).

The instance $(X, \mathcal{R})$ that we are going to construct contains $N \cdot\left(2^{|D|}-1\right)$ variables $x_{A, k}$ for $k=1, \ldots, N$. If $\mathcal{R}_{0}$ contains a constraint $R_{i}=\left(x_{A_{1}}, \ldots, x_{A_{r_{i}}}\right)$ of type $U_{i}$ with arity $r_{i} \geq 2$, then we include the $r_{i}$-tuple of variables $\left(x_{A_{1}, k_{1}}, \ldots, x_{A_{r_{i}}, k_{r_{i}}}\right)$ as a constraint of type $U_{i}$ and weight one to $\mathcal{R}$ with probability equal to $w_{\mathcal{L}}\left(R_{i}\right) N^{1+1 / 2 k-r_{i}}$; constraints $\left(x_{A_{1}, k_{1}}, \ldots, x_{A_{r_{i}}, k_{r_{i}}}\right)$ included to $\mathcal{R}$ because of the constraint $R_{i}$ are said to correspond to $R_{i}$. If $\mathcal{R}_{0}$ contains a constraint $R_{i}=\left(x_{A}\right)$ of type $U_{i}$, then $\mathcal{R}$ contains a constraint $R_{i}=\left(x_{A, k}\right)$ of type $U_{i}$ and weight $w_{\mathcal{L}}\left(R_{i}\right) N^{1 / 2 k}$ with probability one, for $k=1, \ldots, N$.

- Removing short cyles. We next show that, with high probability, it is possibly to remove all cycles of length at most $k$ from the instance $(X, \mathcal{R})$ without significantly decreasing the total weight of all the constraints. Observe now that the instance $(X, \mathcal{R})$ is homomorphic to $\left(X_{0}, \mathcal{R}_{0}\right)$ through the homomorphism $\psi: X \rightarrow X_{0}$ defined as $\psi\left(x_{A, i}\right)=x_{A}$. Hence, the image of a cycle of $(X, \mathcal{R})$ through $\psi$ is a cycle of $\left(X_{0}, \mathcal{R}_{0}\right)$ which is of the same length if we allow repeated constraints.

Fix a cycle $R_{1}, \ldots, R_{\ell}$ of $\left(X_{0}, \mathcal{R}_{0}\right)$ of length $\ell \leq k$. Since $R_{i}$ and $R_{i+1}$ share at least one variable, the number of possible preimages $R_{1}^{\prime}, \ldots, R_{\ell}^{\prime}$ of the cycle $R_{1}, \ldots, R_{\ell}$ is at most $N^{\sum_{i=1}^{\ell} r_{i}-\ell}$ where $r_{i}$ is the arity of $R_{i}$. The probability of including $R_{i}^{\prime}$ to $(X, \mathcal{R})$ is

$$
w_{\mathcal{L}}\left(R_{i}\right) N^{1+1 / 2 k-r_{i}} \leq w_{\mathcal{L}, 0} N^{1+1 / 2 k-r_{i}} .
$$

Hence, the expected number of cycles of $(X, \mathcal{R})$ that are preimages of the cycle $R_{1}, \ldots, R_{\ell}$ is at most

$$
N^{\sum_{i=1}^{\ell} r_{i}-\ell} \cdot \prod_{i=1}^{\ell} w_{\mathcal{L}, 0} N^{1+1 / 2 k-r_{i}}=w_{\mathcal{L}, 0}^{\ell} N^{\ell / 2 k} \leq w_{\mathcal{L}, 0}^{\ell} N^{1 / 2}
$$

If $M$ is the number of constraints of $\mathcal{R}_{0}$, then the number of cycles of $\left(X_{0}, \mathcal{R}_{0}\right)$ of length at most $k$ does not exceed $M^{k}$ and the expected number of cycles of $(X, \mathcal{R})$ is thus at most

$$
M^{k} w_{\mathcal{L}, 0}^{k} N^{1 / 2}
$$


Hence, Markov's inequality (Proposition 2.2) implies that the total weight of constraints of $(X, \mathcal{R})$ contained in a cycle of length at most $k$ is at most (if $N$ is sufficiently large)

$$
2 k M^{k} w_{\mathcal{L}, 0}^{k+1} N^{1 / 2}
$$

with probability at least $1 / 2$. The expression (3.2) is at most

$$
\frac{\varepsilon}{4} w_{\mathcal{L}, 0} N^{1+1 / 2 k}
$$

for $N$ sufficiently large since $k, M, w_{\mathcal{L}, 0}$ and $\epsilon$ do not depend on $N$.

The instance $(X, \mathcal{R})$ without the constraints contained in a cycle of length at most $k$ will be called pruned and denoted by $\left(X^{\prime}, \mathcal{R}^{\prime}\right)$.

- Claim: If $N$ is sufficiently large, then

$$
\operatorname{Prob}\left(w_{0} \geq(1-\varepsilon / 4) w_{\mathcal{L}, 0} N^{1+1 / 2 k}\right) \geq 7 / 8
$$

wher $w_{0}$ is the weight of the constraints of the instance $(X, \mathcal{R})$.

We apply Chernoff's inequality (Proposition 2.3) separately to every set of constraints of $(X, \mathcal{R})$ corresponding to the same constraint $R_{i} \in \mathcal{R}_{0}$ of $2^{\mathcal{L}}$. If $r_{i} \geq 2$ is the arity of $R_{i}$, then there are $N^{r_{i}}$ constraints that can be included to $\mathcal{R}$ and each of them is included with probability $w_{\mathcal{L}}\left(R_{i}\right) N^{1+1 / 2 k-r_{i}}$. Hence, the probability that the total weight of such constraints is less then $(1-$ $\varepsilon / 4) w_{\mathcal{L}}\left(R_{i}\right) N^{1+1 / 2 k}$ is at most

$$
e^{-\frac{\varepsilon^{2} w_{\mathcal{L}}\left(R_{i}\right) N^{1+1 / 2 k}}{32}}
$$

Since there are finitely many constraints $R_{i} \in \mathcal{R}_{0}$, there is with probability at least $7 / 8$ no constraint $R_{i} \in \mathcal{R}_{0}$ such that the weight of constraints corresponding to $R_{i}$ is less than $(1-\varepsilon / 4) w_{\mathcal{L}}\left(R_{i}\right) N^{1+1 / 2 k}$ (if $N$ is sufficiently large). Since $w_{\mathcal{L}, 0}=\sum_{R_{i} \in \mathcal{R}_{0}} w_{\mathcal{L}}\left(R_{i}\right)$, the total weight $w_{0}$ of all the constraints of $(X, \mathcal{R})$ is at least $(1-\varepsilon / 4) w_{\mathcal{L}, 0} N^{1+1 / 2 k}$ with probability at least $7 / 8$. An analogous argument applies to unary constraint types.

- Claim: With probability at least $7 / 8$, every solution $\varphi$ of $(X, \mathcal{R})$ has weight at most $(\pi(\mathcal{L})+\varepsilon / 4) w_{\mathcal{L}, 0} N^{1+1 / 2 k}$.

For simplicity, we first assume that $\mathcal{L}$ does not have unary constraint types. Let us fix a solution $\varphi: X \rightarrow D$ for the rest of this paragraph. We define a function $p: X_{0} \times D \rightarrow[0,1]$ as follows: the value $p\left(x_{A}, d\right)$ is equal to the number of variables $x_{A, k}$ with $\varphi\left(x_{A, k}\right)=d$ divided by $N$. We infer from the construction of $(X, \mathcal{R})$ that for every constraint $R_{i}=\left(x_{A_{1}}, \ldots, x_{A_{r_{i}}}\right) \in \mathcal{R}_{0}$ of type $U_{i} \in \mathcal{U}$ with arity $r_{i} \geq 2$, there are

$$
N^{r_{i}} \sum_{\left(d_{1}, \ldots, d_{r_{i}}\right) \in U_{i}} \prod_{k=1}^{r_{i}} p\left(x_{A_{k}}, d_{k}\right)
$$

constraints satisfied by $\varphi$ that can be included to $(X, \mathcal{R})$. We will call such constraints good. Moreover, we additionally mark $\varepsilon \pi(\mathcal{L}) N^{r_{i}} / 8$ constraints corresponding to $R_{i}$ to be good (if there are not enough additional constraints, mark all constraints corresponding to $R_{i}$ as good-we will point out the case where this exceptional case is handled through the course of our arguments). 
Since each good constraint is included to $(X, \mathcal{R})$ with probability equal to $w_{\mathcal{L}}\left(R_{i}\right) N^{1+1 / 2 k-r_{i}}$, the expected number of good constraints corresponding to $R_{i}$ is

$$
w_{\mathcal{L}}\left(R_{i}\right) N^{1+1 / 2 k}\left(\frac{\varepsilon \pi(\mathcal{L})}{8}+\sum_{\left(d_{1}, \ldots, d_{r_{i}}\right) \in U_{i}} \prod_{k=1}^{r_{i}} p\left(x_{A_{k}}, d_{k}\right)\right) .
$$

Hence, the probability that the number of good constraints corresponding to $R_{i}$ is greater than

$$
(1+\varepsilon / 8) w_{\mathcal{L}}\left(R_{i}\right) N^{1+1 / 2 k}\left(\frac{\varepsilon \pi(\mathcal{L})}{8}+\sum_{\left(d_{1}, \ldots, d_{r_{i}}\right) \in U_{i}} \prod_{k=1}^{r_{i}} p\left(x_{A_{k}}, d_{k}\right)\right)
$$

is at most

$$
e^{-\frac{\varepsilon^{2} w_{\mathcal{L}}\left(R_{i}\right) N^{1+1 / 2 k}\left(\varepsilon \pi(\mathcal{L}) / 8+\sum_{\left(d_{1}, \ldots, d_{r_{i}}\right) \in U_{i}} \Pi_{k=1}^{r_{i}} p\left(x_{A_{k}}, d_{k}\right)\right)}{192}} \leq e^{-\frac{\varepsilon^{3} \pi(\mathcal{L}) w_{\mathcal{L}}\left(R_{i}\right) N^{1+1 / 2 k}}{1536}}
$$

by Chernoff's inequality (Proposition 2.3). In case that there were not additional $\varepsilon \pi(\mathcal{L}) N^{r_{i}} / 8$ constraints to be marked as good, the total number of constraints corresponding to $R_{i}$ is at most

$$
\left(\frac{\varepsilon \pi(\mathcal{L})}{8}+\sum_{\left(d_{1}, \ldots, d_{r_{i}}\right) \in U_{i}} \prod_{k=1}^{r_{i}} p\left(x_{A_{k}}, d_{k}\right)\right) N^{r_{i}}
$$

and the claim follows from the fact that the total weight of all constraints corresponding to $R_{i}$ is greater than (3.3) with probability at most (3.4).

Since the weight function $w_{\mathcal{L}}$ is fixed, the value of $\pi(\mathcal{L})$ is constant independent from the instance, and there are only finitely many constraints in $\mathcal{R}_{0}$, the probability that there exists $R_{i} \in \mathcal{R}_{0}$ such that the number of good constraints corresponding to $R_{i}$ is greater than (3.3) is exponentially small in $N^{1+1 / 2 k}$.

Since there are at most $|D|^{\left(2^{|D|}-1\right) \cdot N}=e^{O(N)}$ choices of $\varphi$, there exists a sufficiently large $N$ such that the number of good constraints corresponding to each $R_{i} \in \mathcal{R}_{0}$ is with probability at least $7 / 8$ (or any other constant smaller than one) bounded from above by (3.3) for all solutions $\varphi$. Summation over all $R_{i} \in \mathcal{R}_{0}$ yields that the total number of satisfied constraints is at most

$$
\begin{gathered}
(1+\varepsilon / 8) N^{1+1 / 2 k} \sum_{R_{i}=\left(x_{1}, \ldots, x_{r_{i}}\right) \in \mathcal{R}_{0}} w_{\mathcal{L}}\left(R_{i}\right) \cdot \sum_{\left(d_{1}, \ldots, d_{r_{i}}\right) \in U_{j}} \prod_{k=1}^{r_{i}} p\left(x_{A_{k}}, d_{k}\right) \\
+(1+\varepsilon / 8) \frac{\varepsilon \pi(\mathcal{L})}{8} N^{1+1 / 2 k} \sum_{R_{i} \in \mathcal{R}_{0}} w_{\mathcal{L}}\left(R_{i}\right)
\end{gathered}
$$

with probability at least $7 / 8$. The definition of $\pi(\mathcal{L})$ and the choice of $w_{\mathcal{L}}$ yield that

$$
\sum_{R_{i}=\left(x_{1}, \ldots, x_{r_{i}}\right) \in \mathcal{R}_{0}} w_{\mathcal{L}}\left(R_{i}\right) \cdot \sum_{\left(d_{1}, \ldots, d_{r_{i}}\right) \in U_{j}} \prod_{k=1}^{r_{i}} p\left(x_{A_{k}}, d_{k}\right) \leq \pi(\mathcal{L}) w_{\mathcal{L}, 0}
$$


which implies that the number of satisfied constraints is at most

$$
\left(1+2 \varepsilon / 8+\varepsilon^{2} / 64\right) N^{1+1 / 2 k} \pi(\mathcal{L}) w_{\mathcal{L}, 0} \leq(1+3 \varepsilon / 8) N^{1+1 / 2 k} \pi(\mathcal{L}) w_{\mathcal{L}, 0}
$$

with probability at least $7 / 8$. An analogous conclusion can be obtained if the language $\mathcal{L}$ might contain unary constraint types.

- Summary. Our previous arguments yield that with probability at least $1 / 4$, the weight of the optimum solution of $(X, \mathcal{R})$ is at most

$$
(1+3 \varepsilon / 8) N^{1+1 / 2 k} \pi(\mathcal{L}) w_{\mathcal{L}, 0},
$$

the total weight $w_{0}$ of the constraints of $(X, \mathcal{R})$ is at least

$$
(1-\varepsilon / 4) N^{1+1 / 2 k} w_{\mathcal{L}, 0}
$$

and the total weight of constraints removed because of their containment in cycles of length at most $k$ is at most

$$
\frac{\varepsilon}{4} w_{\mathcal{L}, 0} N^{1+1 / 2 k}
$$

which implies that the weight $w_{0}^{\prime}$ of the pruned instance $\left(X^{\prime}, \mathcal{R}^{\prime}\right)$ is at least

$$
(1-\varepsilon / 2) N^{1+1 / 2 k} w_{\mathcal{L}, 0} .
$$

Hence, with probability at least $1 / 4$, the weight of an optimum solution of $\left(X^{\prime}, \mathcal{R}^{\prime}\right)$ is at most

$$
\frac{1+3 \varepsilon / 8}{1-\varepsilon / 2} \pi(\mathcal{L}) w_{0}^{\prime} \leq(1+\varepsilon) \pi(\mathcal{L}) w_{0}^{\prime} .
$$

This inequality holds since we have assumed that $\varepsilon<1 / 10$. Because the instance $\left(X^{\prime}, \mathcal{R}^{\prime}\right)$ does not contain a cycle of length at most $k$ and is homomorphic to $2^{\mathcal{L}}$, it is vertex and constraint $k$-consistent by Lemma 3.2.

\section{口}

4. Algorithmic Results. In this section, we design our linear-time algorithm for a fixed constraint language $\mathcal{L}$, and prove the lower bounds on the limits.

ThEOREM 4.1. Let $\mathcal{L}=(D, \mathcal{U})$ be a fixed constraint language. There exists an algorithm that for an input instance $(X, \mathcal{R})$ with total weight $w_{0}$ and a given real number $\varepsilon>0$,

- either constructs a solution $\varphi$ of weight $(\pi(\mathcal{L})-\varepsilon) w_{0}$, or

- finds a set of at most $f(\varepsilon)$ constraints of $\mathcal{R}$ that cannot be simultaneously satisfied

where $f(\varepsilon)$ is a function that only depends on $\mathcal{L}$ and $\varepsilon$. The running time of the algorithm is linear in $|X|+|\mathcal{R}|$ and polynomial in $1 / \varepsilon$ (for a fixed constraint language $\mathcal{L})$.

Proof. Let us briefly describe the main steps of the algorithm and then focus on each step separately. The parameter $r$ used in the description of the algorithm is the maximum arity of a constraint of $\mathcal{R}$.

1. First, we construct sets $\mathcal{R}_{i} \subseteq \mathcal{R}, i=1, \ldots, 2|D| r / \varepsilon$, such that the instance $\left(X, \mathcal{R} \backslash \mathcal{R}_{i}\right)$ is homomorphic to $2^{\mathcal{L}}$ for every $i$.

2. If the algorithm fails to construct the sets, then it exhibits a set of at most $f(\varepsilon)$ inconsistent constraints. 
3. If the algorithm does not fail to construct the sets $\mathcal{R}_{i}$, we remove the set $\mathcal{R}_{i}$ with the least weight from the input instance. We show that this decreases the total weight of the instance by at most $\varepsilon w_{0} / 2$.

4. We next compute a function $p: X \times D \rightarrow[0,1]$ such that if $x \in X$ is assigned a value $d \in D$ with probability $p(x, d)$, then the expected weight of satisfied constraints is at least $(\pi(\mathcal{L})-\varepsilon) w_{0}$.

5. Finally, using standard derandomization techniques, we find a solution $\varphi$ : $X \rightarrow D$ of weight at least $(\pi(\mathcal{L})-\varepsilon) w_{0}$.

Here and hereafter, we assume for simplicity that $\varepsilon$ is the inverse of an integer.

We now proceed with the description of the algorithm.

1. Construction of the sets $\mathcal{R}_{i}$. In addition to the sets $\mathcal{R}_{i} \subseteq \mathcal{R}$, we construct functions $\psi_{i}: X \rightarrow 2^{D}$ such that the mapping that assigns $x \in X$ the variable $x_{\psi_{i}(x)}$ of $2^{\mathcal{L}}$ is a homomorphism from $\left(X, \mathcal{R} \backslash \mathcal{R}_{i}\right)$ to $2^{\mathcal{L}}$. Together with the mapping $\psi_{i+1}$ we construct a mapping $\Psi_{i}: X \rightarrow 2^{\mathcal{R}}$ which assigns to a variable $x$ a subset of the input contraints forcing its value to be one of those contained in $\psi_{i}(x)$. The mapping $\psi_{i+1}$ and $\Psi_{i+1}$ will be constructed at the stage when the set $\mathcal{R}_{i}$ is constructed.

Initially, $\psi_{1}(x)=D$ and $\Psi_{1}(x)=\emptyset$ for every $x \in X$. Assume that the sets $\mathcal{R}_{1}, \ldots, \mathcal{R}_{i-1}$ and mappings $\psi_{1}, \ldots, \psi_{i}$ and $\Psi_{1}, \ldots, \Psi_{i}$ have been constructed We now construct the set $\mathcal{R}_{i}$ and the mappings $\psi_{i+1}$ and $\Psi_{i+1}$.

- Set $\psi_{i+1}(x)$ to $\psi_{i}(x)$ and $\Psi_{i+1}(x)$ to $\Psi_{i}(x)$ for every $x \in X$.

- As long as there exists a constraint $\left(x_{1}, \ldots, x_{k}\right)$ in $\mathcal{R}$ with type $U_{j} \in \mathcal{U}$, an index $j^{\prime}$ and a value $d_{j^{\prime}} \in \psi_{i+1}\left(x_{j^{\prime}}\right)$ such that

$$
\psi_{i}\left(x_{1}\right) \times \cdots \times \psi_{i}\left(x_{j^{\prime}-1}\right) \times\left\{d_{j^{\prime}}\right\} \times \psi_{i}\left(x_{j+1}\right) \times \cdots \times \psi_{i}\left(x_{k}\right) \cap U_{j}=\emptyset,
$$

i.e., there is no choice of $d_{j^{\prime \prime}} \in \psi_{i}\left(x_{j^{\prime \prime}}\right)$ for $j^{\prime \prime}=1, \ldots, j^{\prime}-1, j^{\prime}+1, \ldots, k$ with $\left(d_{1}, \ldots, d_{k}\right) \in U_{j}$, proceed as follows:

- remove $d_{j^{\prime}}$ from $\psi_{i+1}\left(x_{j^{\prime}}\right)$ and

- add to $\Psi_{i+1}\left(x_{j^{\prime}}\right)$ the union

$$
\left\{\left(x_{1}, \ldots, x_{k}\right)\right\} \cup \bigcup_{1 \leq m \leq k, m \neq j^{\prime}} \Psi_{i}\left(x_{m}\right)
$$

where the type of the constraint $\left(x_{1}, \ldots, x_{k}\right)$ is $U_{j}$.

- Set $\mathcal{R}_{i}$ to be the set of constraints that are not mapped by $\psi_{i}$ to a constraint of $2^{\mathcal{L}}$.

Clearly, $\psi_{i}$ is a homomorphism from $\left(X, \mathcal{R} \backslash \mathcal{R}_{i}\right)$ to $2^{\mathcal{L}}$ unless $\psi_{i}(x)=\emptyset$ for some $x$. Note that if a constraint $\left(x_{1}, \ldots, x_{k}\right)$ of type $U_{j}$ is contained in $\mathcal{R}_{i}$, then there exists index $j^{\prime}$ and a value $d_{j^{\prime}} \in \psi_{i}\left(x_{j^{\prime}}\right)$ with the properties given above. In particular, $d_{j^{\prime}} \notin \psi_{i+1}\left(x_{j^{\prime}}\right)$ but the removal of $d_{j^{\prime}}$ from $\psi_{i+1}\left(x_{j^{\prime}}\right)$ could be caused by a different constraint which also forbids $d_{j^{\prime}}$ from being assigned to $x_{j^{\prime}}$.

We now show that the constraints contained in $\Psi_{i}\left(x_{j}\right)$ force the value of a variable $x_{j}$ to be one of the values contained in $\psi_{i}\left(x_{j}\right)$, i.e., any exact solution of the instance $\left(X, \Psi_{i}\left(x_{j}\right)\right)$ assigns $x_{j}$ a value contained in $\psi_{i}\left(x_{j}\right)$. The proof is by induction on $i$. If $i=1$, there is nothing to prove since $\psi_{1}\left(x_{j}\right)=D$. For $i>1$, if $\psi_{i}\left(x_{j}\right)=\psi_{i-1}\left(x_{j}\right)$, then $\Psi_{i}\left(x_{j}\right)=\Psi_{i-1}\left(x_{j}\right)$ and the claim follows by induction. If $\psi_{i}\left(x_{j}\right) \subset \psi_{i-1}\left(x_{j}\right)$, then for every $d_{j} \in \psi_{i-1}\left(x_{j}\right) \backslash \psi_{i}\left(x_{j}\right)$, there is a constraint $\left(x_{1}, \ldots, x_{k}\right) \in \mathcal{R}$ that caused the value $d_{j}$ to be removed. The constraints contained in the sets 
$\Psi_{i-1}\left(x_{1}\right), \ldots, \Psi_{i-1}\left(x_{j-1}\right), \Psi_{i-1}\left(x_{j+1}\right), \ldots, \Psi_{i-1}\left(x_{k}\right)$ force the values of the variables $x_{1}, \ldots, x_{j-1}, x_{j+1}, \ldots, x_{k}$ to be only those contained in $\psi_{i-1}\left(x_{1}\right)$, $\ldots, \psi_{i-1}\left(x_{j-1}\right), \psi_{i-1}\left(x_{j+1}\right), \ldots, \psi_{i-1}\left(x_{k}\right)$. Since this is incompatible with assigning the value $d_{j}$ to $x_{j}$, the constraints contained in $\Psi_{i-1}\left(x_{1}\right) \cup \cdots \cup$ $\Psi_{i-1}\left(x_{j-1}\right) \cup \Psi_{i-1}\left(x_{j+1}\right) \cup \cdots \cup \Psi_{i-1}\left(x_{k}\right)$ and the constraint $\left(x_{1}, \ldots, x_{k}\right)$ forces that $x_{j}$ cannot be assigned $d_{j}$.

2. Identifying an inconsistent set of constraints.

If there exist $i$ and $x$ such that $\psi_{i}(x)=\emptyset$, then the constraints contained in $\Psi_{i}(x)$ restrict the values of $x$ to those of $\psi_{i}(x)=\emptyset$, i.e., the subinstance $\left(X, \Psi_{i}(x)\right)$ has no exact solution. The algorithm returns the set $\Psi_{i}(x)$ of inconsistent constraints - next, we bound its size.

We prove that a set $\Psi_{i}(x)$ contains at most $(|D| r+1)^{i}$ constraints by induction on $i$. The bound clearly holds for $i=1$ since the sets $\Psi_{1}(x)$ are empty. Each set $\Psi_{i}(x)$ is a union of at most $|D| r$ different sets $\Psi_{i-1}\left(x^{\prime}\right)$ with at most $|D|$ additional constraints, i.e., its size does not exceed

$$
\left|\Psi_{i}(x)\right| \leq|D|+|D| r(|D| r+1)^{i-1} \leq(|D| r+1)^{i} .
$$

Hence, the size of the set of inconsistent constraints returned by the algorithm does not exceed $(|D| r+1)^{2|D| r / \varepsilon}$, which is a function that only depends on $\varepsilon$ (recall that the constraint language $\mathcal{L}$ is fixed). Let us remark that the bound on the size of the sets of inconsistent constraints can be substantially decreased by a finer analysis. In the actual implementation of this step of the algorithm, we do not have to compute the sets $\Psi_{i}(x)$ explicitly, however, for each removal of a value $d \in D$ from $\psi_{i}(x)$, we mark which of the input constraints caused the removal of $d$ and compute the set $\Psi_{i}(x)$ (in linear time in the input size) only when the set is supposed to be output.

3. Finding a small set of constraints to be removed from the input instance.

Suppose now that the algorithm does not output an unsatisfiable set of constraints, i.e., $\psi_{i}(x) \neq \emptyset$ for all $i=1, \ldots, 2|D| r / \varepsilon$ and $x \in X$. Observe that each constraint $R_{j} \in \mathcal{R}$ is contained in at most $|D| r$ sets $\mathcal{R}_{i}$ : each time a constraint $R_{j}$ is included into a set $\mathcal{R}_{i}$, the cardinality of the set $\psi_{i}(x)$ of one of the variables $x$ contained in $R_{j}$ is decreased by one. Since $R_{j}$ contains at most $r$ variables and the sets $\psi_{1}(x)$ contain $|D|$ elements each, $R_{j}$ is included in at most $|D| r$ sets $\mathcal{R}_{j}$. Hence, the total sum of weights of constraints contained in all the sets $\mathcal{R}_{i}$ is at most $|D| r w_{0}$. Since there are $2|D| r / \varepsilon$ sets $\mathcal{R}_{i}$, there exists a set $\mathcal{R}_{i_{0}}$ such that the sum of the weights of the constraints contained in $\mathcal{R}_{i_{0}}$ is at most $\varepsilon w_{0} / 2$. Fix such an index $i_{0}$ for the rest of the proof.

4. Computing a good probability destribution.

By the construction, the instance $\left(X, \mathcal{R} \backslash \mathcal{R}_{i_{0}}\right)$ is homomorphic to $2^{\mathcal{L}}$ and the homomorphism maps a variable $x \in X$ to $x_{A}$ for $A=\psi_{i_{0}}(x)$. Next, we define values $p(A, d)$ for non-empty subsets $A \subseteq D$ and $d \in D$ such that $\sum_{d \in D} p(A, d)=1$ for every $A$. Let $E(p)$ be the expected weight of the satisfied constraints if each variable $x \in X$ is assigned randomly and independently a value $d \in D$ with probability $p\left(\psi_{i_{0}}(x), d\right)$. Let $E_{0}$ be the maximum value of $E$ under the restrictions that $0 \leq p(A, d) \leq 1$ and $\sum_{d \in D} p(A, d)=1$. Since the number of variables on which the function $E(p(A, d))$ depends is finite, it is possible to find, in time polynomial in $1 / \varepsilon$, a function $p_{0}$ such that $E\left(p_{0}\right)$ 
is at least $(1-\varepsilon / 2) E_{0}$. By the definition of $\pi(\mathcal{L})$, we have that $E_{0} \geq \pi(\mathcal{L}) w_{0}^{\prime}$ where $w_{0}^{\prime}$ is the total weight of constraints of $\left(X, \mathcal{R} \backslash \mathcal{R}_{i_{0}}\right)$. Hence, the value of $E\left(p_{0}\right)$ is at least

$$
(1-\varepsilon / 2) \pi(\mathcal{L}) w_{0}^{\prime} \geq(1-\varepsilon / 2)^{2} \pi(\mathcal{L}) w_{0} \geq(1-\varepsilon) \pi(\mathcal{L}) w_{0} \geq(\pi(\mathcal{L})-\varepsilon) w_{0} .
$$

In particular, if the value of each variable $x \in X$ is set to be $d \in D$ with probability $p_{0}\left(\psi_{i_{0}}(x), d\right)$, then the expected weight of satisfied constraints is at least $(\pi(\mathcal{L})-\varepsilon) w_{0}$.

5. Derandomization.

The final stage of the algorithm (once the function $p_{0}$ has been constructed) lies in derandomizing the choices of values of the variables $x \in X$. A standard technique of derandomization using conditional expectations can be applied in this scenario. We are not going to describe the details of the entire derandomization process and refer to standard literature on the subject, e.g., [27]. Let us just remark that the derandomization can be done in time linear in the size of the input.

There is an immediate corollary of Theorems 3.4 and 4.1.

Corollary 4.2. Let $\mathcal{L}=(D, \mathcal{U})$ be a constraint language. Then the following equalities hold.

$$
\rho_{v}^{w}(\mathcal{L})=\rho_{c}^{w}(\mathcal{L})=\pi(\mathcal{L})
$$

If $\mathcal{L}$ does not contain unary constraint types, then

$$
\rho_{v}(\mathcal{L})=\rho_{c}(\mathcal{L})=\pi(\mathcal{L}) .
$$

5. Unweighted and Weighted Instances. We have seen that $\rho_{v}^{w}(\mathcal{L})=\rho_{v}(\mathcal{L})$ and $\rho_{c}^{w}(\mathcal{L})=\rho_{c}(\mathcal{L})$ if the constraint language $\mathcal{L}$ does not contain unary constraint types. It turns out that this is not just a coincidence, but the following stronger statement holds. The same idea that we use in the following proof has been used before in the paper of Crescenzi, Silvestri and Trevisan [4] to design reductions between weighted and unweighted versions of several optimization problems.

THEOREM 5.1. Let $\mathcal{L}=(D, \mathcal{U})$ be a fixed constraint language without unary constraint types. For every $k \geq 1$, the following holds:

$$
\rho_{v, k}^{w}(\mathcal{L})=\rho_{v, k}(\mathcal{L}) \text { and } \rho_{c, k}^{w}(\mathcal{L})=\rho_{c, k}(\mathcal{L}) .
$$

Proof. We focus on proving that $\rho_{v, k}^{w}(\mathcal{L})=\rho_{v, k}(\mathcal{L})$; the proof for the constraint $k$-consistent instances is analogous. Since it is obvious that $\rho_{v, k}^{w}(\mathcal{L}) \leq \rho_{v, k}(\mathcal{L})$, we have to show that $\rho_{v, k}^{w}(\mathcal{L}) \geq \rho_{v, k}(\mathcal{L})$. Fix $\varepsilon>0$. By the definition of $\rho_{v, k}^{w}(\mathcal{L})$, there exists a weighted variable $k$-consistent instance $\left(X_{0}, \mathcal{R}_{0}\right)$ with the weight function $w$ that does not have solution of weight greater than $\left(\rho_{v, k}^{w}(\mathcal{L})+\varepsilon / 2\right) w_{0}$ where $w_{0}$ is the total weight of the constraints of $\left(X_{0}, \mathcal{R}_{0}\right)$. Our goal is to construct an unweighted instance $(X, \mathcal{R})$ with some number $m$ of constraints that does not have a solution satisfying more than $\left(\rho_{v, k}^{w}(\mathcal{L})-\varepsilon\right) m$ of its constraints. Since the construction is similar to the one presented in the proof of Theorem 3.4, we decided to provide all the details only where the two proofs differ, and sketch the arguments where they are analogous. 
Let $X_{0}=\left\{x_{1}, \ldots, x_{n}\right\}$ and let $N$ be a sufficiently large integer. The instance $(X, \mathcal{R})$ contains $n \cdot N$ variables $x_{i, j}, 1 \leq i \leq n$ and $1 \leq j \leq N$. For every constraint $R_{k}=\left(x_{i_{1}}, \ldots, x_{i_{r}}\right) \in \mathcal{R}$ of type $U_{k}$ with $r^{\prime} \leq r$ distinct variables, a constraint $\left(x_{i_{1}, j_{1}}, \ldots, x_{i_{r}, j_{r}}\right)$ is included to $\mathcal{R}$ with probability $w\left(R_{k}\right) N^{2-r^{\prime}}$; we require that if a single variable, e.g., $x_{i_{1}}$ appears several times in $R_{k}$, then all its occurrences are replaced by the same variable $x_{i_{1}, j_{1}}$.

Observe that the constructed instance $(X, \mathcal{R})$ is homomorphic to the original instance $\left(X_{0}, \mathcal{R}_{0}\right)$ (just map a variable $x_{i, j} \in X$ to $\left.x_{i} \in X_{0}\right)$. Hence, if $X^{\prime} \subseteq X$ is a set of at most $k$ variables of $X$, the subinstance $\left(X^{\prime}, \mathcal{R}^{\prime}\right)$ of $(X, \mathcal{R})$ where $\mathcal{R}^{\prime}$ are those constraints of $\mathcal{R}$ that contain only the variables of $X^{\prime}$, is homomorphic to a subinstance of $\left(X_{0}, \mathcal{R}_{0}\right)$ with at most $k$ variables. Since the subinstance of $\left(X_{0}, \mathcal{R}_{0}\right)$ has an exact solution, the subinstance $\left(X^{\prime}, \mathcal{R}^{\prime}\right)$ has also an exact solution. In particular, the instance $(X, \mathcal{R})$ is variable $k$-consistent.

An application of Chernoff's bound (Proposition 2.3) yields that with probability at least $3 / 4$ the number of constraints of $(X, \mathcal{R})$ is at least $(1-\varepsilon / 8) w_{0} \cdot N^{2}$. The details of the proof are analogous to the proof of Theorem 3.4.

Fix one out of the $|D|^{n N}$ possible solutions $\varphi: X \rightarrow D$ of $(X, \mathcal{R})$. Let $p\left(x_{i}, d\right)$ for $x_{i} \in X_{0}$ and $d \in D$ be the size $\varphi^{-1}(d) \cap\left\{x_{i, 1}, \ldots, x_{i, N}\right\}$ divided by $N$. By the choice of $\left(X_{0}, \mathcal{R}_{0}\right)$, the expected weight of constraints of $\mathcal{R}_{0}$ that are satisfied when each variable $x_{i} \in X_{0}$ is assigned randomly (and independently of other variables of $X_{0}$ ) a value $d \in D$ with probability $p\left(x_{i}, d\right)$ is at most the weight of an optimum solution, which is at most $\left(\rho_{v, k}^{w}(\mathcal{L})+\varepsilon / 2\right) w_{0}$. If $p$ is the probability that a constraint $R_{k} \in \mathcal{R}_{0}$ is satisfied, there are $p N^{r^{\prime}}$ constraints $R$ corresponding to $R_{k}$ that can be included to $\mathcal{R}$ and that are satisfied by $\varphi$.

Another application of Chernoff's bound yields that with probability at most $e^{-\Theta\left(N^{2}\right)}$, the number of constraints corresponding to $R_{k}$ that are satisfied by $\varphi$ and included to $\mathcal{R}$ is greater than $(1+\varepsilon / 8) p w\left(R_{k}\right) N^{2}$. Hence, the total number of constraints satisfied by $\varphi$ is greater than $(1+\varepsilon / 8)\left(\rho_{v, k}^{w}(\mathcal{L})+\varepsilon / 2\right) w_{0} N^{2}$ with probability at most $e^{-\Theta\left(N^{2}\right)}$. Since there are $|D|^{n N}$ choices of $\varphi$, the total number of constraints satisfied by any solution $\varphi$, is at most $(1+\varepsilon / 8)\left(\rho_{v, k}^{w}+\varepsilon / 2\right) w_{0} N^{2}$ with probability at most $7 / 8$ if $N$ is sufficiently large.

We conclude that there exists a variable $k$-consistent instance $(X, \mathcal{R})$ with at least $(1-\varepsilon / 8) w_{0} N^{2}$ constraints and the number of constraints that can be satisfied by a solution $\varphi$ is at most

$$
(1+\varepsilon / 8)\left(\rho_{v, k}^{w}+\varepsilon / 2\right) w_{0} N^{2} \leq\left(\rho_{v, k}^{w}+\varepsilon\right) m
$$

where $m$ is the number of constraints of $(X, \mathcal{R})$. Since such an instance exists for every $\varepsilon>0$, it follows that $\rho_{v, k} \leq \rho_{v, k}^{w}$.

Let us comment on one difference between the proof of Theorem 3.4 and the proof of Theorem 5.1: in the former proof, we needed to prune the random instance to remove constraints that contain the same variable several times. Such constraints could spoil the acyclicity of the constructed instance and thus its consistency. However, in the latter proof, the constructed instance is always homomorphic to a locally consistent instance $\left(X_{0}, \mathcal{R}_{0}\right)$ and thus locally consistent, too. Such an argument cannot be used in the former proof, since $2^{\mathcal{L}}$ need not be locally consistent.

6. Graph Homomorphisms. In this section, we discuss how our results on general CSPs relate to the results obtained in our conference paper [1] on CSPs corresponding to graph homomorphisms. In the following, we state all the results 
for directed graphs. Analogous results for undirected graphs follow directly from the results on directed graphs. As discussed in Section 2, the corresponding CSPs have a constraint language that contains a single binary constraint type. Throughout this section, $\mathcal{L}_{H}$ stands for the constraint language obtained from a (directed) graph $H$ through the construction described in Section 2.

One of the results in [1] is that $\rho_{v}\left(\mathcal{L}_{H}\right)=\rho_{c}\left(\mathcal{L}_{H}\right)=1$ if and only if the graph $H$ has tree duality. A graph $H$ has tree duality iff a graph $G$ is homomorphic to $H$ when every directed tree homomorphic to $G$ is also homomorphic to $H$. Examples of graphs with tree duality include orientations of paths or acyclic tournaments. The set structure $2^{H}$ of a graph $H$ is again a graph, and is called the set graph of $H$. An equivalent characterization of tree duality asserts that a directed graph $H$ has tree duality if and only if its set graph $2^{H}$ is homomorphic to $H[5,11]$.

The following is then an immediate consequence of Corollary 4.2 .

TheOREM 6.1. Let $H$ be a directed graph. The equalities

$$
\rho_{v}\left(\mathcal{L}_{H}\right)=1 \text { and } \rho_{c}\left(\mathcal{L}_{H}\right)=1
$$

hold if and only if $H$ has tree duality.

The other types of CSP problems addressed in [1] were those corresponding to graphs $H$ with a directed cycle. For such a constraint language $\mathcal{L}_{H}$, we relate $\pi\left(\mathcal{L}_{H}\right)$ the quantity called fractional relative density. The fractional relative density $\delta_{\text {rel }}^{\prime}(H)$ of $H$ is defined as

$$
\delta_{\text {rel }}^{\prime}(H)=\max _{p: V(H) \rightarrow[0,1]} \sum_{u v \in E(H)} p(u) \cdot p(v)
$$

where the maximum is taken over all functions $p: V(H) \rightarrow[0,1]$ such that the sum of $p(v)$ is equal to one. Let us state the relation between $\mathcal{L}_{H}$ and $\delta_{\text {rel }}^{\prime}(H)$ formally:

Proposition 6.2. Let $H$ be a directed graph containing a directed cycle and let $\mathcal{L}_{H}$ be the corresponding constraint language. The quantity $\pi\left(\mathcal{L}_{H}\right)$ is equal to the fractional relative density $\delta_{\text {rel }}^{\prime}(H)$ of $H$.

Proof. Let $W$ be the vertex set of a directed cycle of $H$. Observe that the set instance $2^{\mathcal{L}_{H}}$ contains a constraint $\left(x_{W}, x_{W}\right)$ (in a graph theory notation, the set graph $2^{H}$ contains a loop at the vertex $x_{W}$ ), and consider the weight function $w$ that assigns weight one to $\left(x_{W}, x_{W}\right)$ and zero weight to the remaining constraints of $2^{\mathcal{L}_{H}}$. For the weight function $w$, the expression that is maximized in (3.1) is equal $\delta_{\text {rel }}^{\prime}(H)$. This implies that $\pi\left(\mathcal{L}_{H}\right) \leq \delta_{\text {rel }}^{\prime}(H)$.

For the other inequality, consider a weight function $w$ minimizing (3.1). Let $p_{0}$ be the function maximizing $(6.1)$ and $p(x, v)=p_{0}(v)$ for every variable $x$ of $2^{\mathcal{L}_{H}}$ and every vertex $v$ of $H$. By the choice of $p_{0}$ and $p$, the inner sum in (3.1) for $p$ is equal to $(6.1)$, i.e., to $\delta_{\text {rel }}^{\prime}(H)$, for every constraint of $2^{\mathcal{L}_{H}}$. Since the total weight of $w$ is equal to one, the whole sum in $(3.1)$ is equal to $\delta_{\text {rel }}^{\prime}(H)$. We conclude that $\pi\left(\mathcal{L}_{H}\right) \geq \delta_{\text {rel }}^{\prime}(H)$ which finishes the proof.

The following result from [1] now follows from Proposition 6.2 and Corollary 4.2:

THEOREM 6.3. If $H$ is a directed graph that contains at least one directed cycle, then

$$
\rho_{v}\left(\mathcal{L}_{H}\right)=\rho_{c}\left(\mathcal{L}_{H}\right)=\delta_{\text {rel }}^{\prime}(H)
$$


If the constraint language $\mathcal{L}_{H}$ consists of a single symmetric binary relation, i.e., the graph $H$ contains an arc $u v$ for every arc $v u$ and vice versa, then the following holds (note that such languages correspond to undirected graphs).

COROLlaRY 6.4. If $H$ is a symmetric directed graph, then

$$
\rho_{v}\left(\mathcal{L}_{H}\right)=\rho_{c}\left(\mathcal{L}_{H}\right)=1
$$

if $H$ contains a loop, and otherwise

$$
\rho_{v}\left(\mathcal{L}_{H}\right)=\rho_{c}\left(\mathcal{L}_{H}\right)=1-1 / \omega
$$

where $\omega$ is the order of the largest clique of $H$.

Proof. By Theorem 6.3, it is enough to show that $\delta_{\text {rel }}^{\prime}(H)=1$ if $H$ has a loop and $\delta_{\text {rel }}^{\prime}(H)=1-1 / \omega$, otherwise. Clearly, $\delta_{\text {rel }}^{\prime}(H) \leq 1$. If $H$ has a loop at a vertex $v$, then the function $p$ that is equal to 1 for $v$ and 0 otherwise witnesses that $\delta_{\text {rel }}^{\prime}(H)=1$.

We now assume that $H$ is loopless. Let $p$ be the function that maximizes (6.1) and among all such functions the one with minimal support. If the support of $p$ induces a clique of order $\omega$, then (6.1) is maximized if $p$ is equal to $1 / \omega$ for the vertices of the clique. If the support of $p$ does not induce a clique, then there are two nonadjacent vertices $u$ and $v$ with $p(u)>0$ and $p(v)>0$. Let $P_{u}=\sum_{u w \in E(H)} p(w)$ and $P_{v}=\sum_{u w \in E(H)} p(w)$. By symmetry, we can assume that $P_{u} \leq P_{v}$. Consider the following function $p^{\prime}$ :

$$
p^{\prime}(w)=\left\{\begin{array}{cl}
p(u)-\min \{p(u), p(v)\} & \text { if } w=u, \\
p(v)+\min \{p(u), p(v)\} & \text { if } w=v, \text { and } \\
p(w) & \text { otherwise. }
\end{array}\right.
$$

Since the vertices $u$ and $v$ are non-adjacent in $H$, the following holds:

$$
\sum_{u v \in E(H)} p^{\prime}(u) \cdot p^{\prime}(v)-\sum_{u v \in E(H)} p(u) \cdot p(v)=2\left(P_{v}-P_{u}\right) \min \{p(u), p(v)\} .
$$

The choice of $p$ implies that $P_{u}=P_{v}$ and $\delta_{\text {rel }}^{\prime}(H)=\sum_{u v \in E(H)} p^{\prime}(u) \cdot p^{\prime}(v)$. Since $P_{u}=P_{v}$, the configuration is again symmetric with respect to $u$ and $v$ and we may assume that $p(u)<p(v)$. Consequently, $p^{\prime}(u)=0$ which contradicts the choice of $p$ as the function maximizing (6.1) that has minimal support.

7. Concluding Remarks. It is natural to ask whether Expression (3.1) in Definition 3.3 can be simplified. It does not seem to be the case. The maximization over all functions $p$, the innermost sum and the product correspond to the maximization, the sum and the product in Expression (6.1) of relative density, respectively. In [1], we discussed that the definition of relative density does not seem to be replaceable by a simpler concept (e.g., by the relative density as defined in [19]). It also seems that the weight function $w$ cannot be avoided, because this function allows us to distinguish the relevant part of the set structure $2^{\mathcal{L}}$.

An issue that we were not able to settle is whether the equalities $\rho_{v}(\mathcal{L})=\rho_{v}^{w}(\mathcal{L})$ and $\rho_{c}(\mathcal{L})=\rho_{c}^{w}(\mathcal{L})$ also hold for constraint languages that contain unary constraints. Under the assumption that no repetitions of constraints in instances are allowed (which is a reasonable assumption since otherwise we can simulate the constraint weights by including the same constraint into an instance several times), we are not aware of any partial result in this direction. We conjecture that the equalities $\rho_{v}^{w}(\mathcal{L})=\rho_{v}(\mathcal{L})$ and $\rho_{c}^{w}(\mathcal{L})=\rho_{c}(\mathcal{L})$ do not hold in general. 
We would also like to make a few remarks on constraint languages with an infinite number of constraint types (but a finite domain). Such constraint languages include in particular CNF formulas with unbounded clause size (clauses are viewed as constraints). Theorems 3.4 and 5.1 translate smoothly to this setting. In (3.1), we replace the minimum with the infimum and require the weight function $w$ to be non-zero for only a finite number of constraints of $\mathcal{R}$. Note that the maximum in (3.1) is always attained. If $\pi(\mathcal{L})$ is defined in this way, the proofs of Theorems 3.4 and 5.1 can be altered as follows. We consider the weight function $w_{\mathcal{L}}$ that is $\varepsilon$-close to $\pi(\mathcal{L})$ instead of that which minimizes (3.1), and construct a $k$-consistent instance with weight $w_{0}^{\prime}$ and with an optimum solution of weight at most $(1+\varepsilon) 2 \pi(\mathcal{L}) w_{0}^{\prime}$. This yields the statements of both Theorems 3.4 and 5.1. On the other hand, we do not know whether Theorem 4.1 holds in this setting, too. The main obstacle is that the bound on the size of a set of inconsistent constraints involves the maximum arity of a constraint of an input instance, which may not be bounded.

Acknowledgments.. The authors would like to thank Pavol Hell for his comments on tree duality and related notions. The authors would also like to thank both the referees for their valuable comments which resulted to a significant improvement of the presentation of the results contained in the paper.

\section{REFERENCES}

[1] M. Bodirsky, D. Král': Locally Consistent Constraint Satisfaction Problems with Binary Constraints, In: Proc. 31th Workshop on Graph-Theoretic Concepts in Computer Science (WG'05), LNCS vol. 3787, Springer-Verlag Berlin (2005), 295-306.

[2] A. Bulatov, A. Krokhin, P. G. Jeavons: Classifying the complexity of constraints using finite algebras. SIAM Journal on Computing 34 (2005), 720-742.

[3] S. Cook, D. Mitchell: Finding Hard Instances of the Satisfiability Problem: A Survey. In: The Satisfiability Problem: Theory and Applications. DIMACS Series in DMTCS Vol. 35 AMS (1997).

[4] P. Crescenzi, R. Silvestri, L. Trevisan: On Weighted vs Unweighted Versions of Combinatorial Optimization Problems. Inf. Comput. 167(1) (2001), 10-26.

[5] V. Dalmau, J. Pearson: Closure Functions and Width 1 Problems. In: Proc. 5th International Conferences on Principles and Practice of Constraint Programming (CP), LNCS Vol. 1713, Springer-Verlag Berlin (1999) 159-173.

[6] R. Dechter, P. van Beek: Local and Global Relational Consistency. Theor. Comput. Sci. 173 (1997) 283-308.

[7] Rina Dechter, Judea Pearl: Tree Clustering for Constraint Networks. Artif. Intell. 38(3) (1989) $353-366$.

[8] Z. Dvořák, D. Král', O. Pangrác: Locally Consistent Constraint Satisfaction Problems, Theor. Comput. Sci 348 (2005), 187-206. A preliminary version appeared in: Proc. 31st International Colloquium on Automata, Languages and Programming (ICALP), LNCS Vol. 3142, Springer-Verlag Berlin (2004), 469-480.

[9] D. Eppstein: Improved Algorithms for 3-coloring, 3-edge-coloring and Constraint Satisfaction. In: Proc. 12th ACM-SIAM Symposium on Discrete Algorithms, SODA (2001) 329-337.

[10] T. Feder, R. Motwani: Worst-case Time Bounds for Coloring and Satisfiability Problems. J. Algorithms 45(2) (2002) 192-201.

[11] T. Feder, M. Vardi: Monotone monadic SNP and constraint satisfaction. In: Proc. 25th Symposium on the Theory of Computation, STOC (1993) 612-622.

[12] T. Feder, M. Vardi: The computational structure of monotone monadic SNP and constraint satisfaction: A study through Datalog and group theory, SIAM Journal on Computing 28 (1999), 57-104.

[13] E. C. Freuder: A sufficient condition for backtrack-free search. J. ACM 29 (1982) 24-32.

[14] Eugene C. Freuder: Complexity of K-Tree Structured Constraint Satisfaction Problems. AAAI 1990: 4-9.

[15] T. Hagerup, Ch. Rüb: A guided tour Chernoff bounds. Inform. Process. Letters 33 (1989) 305-308

[16] P. Hell, J. Nešetřil: Graphs and Homomorphisms. Oxford University Press, 2004. 
[17] P. Hell, J. Nešetřil, X. Zhu: Duality and polynomial testing of tree homomorphisms. Trans. Amer. Math. Soc. 348(4) (1996) 1281-1297.

[18] M. A. Huang, K. Lieberherr: Implications of Forbidden Structures for Extremal Algorithmic Problems. Theor. Comput. Sci. 40 (1985) 195-210.

[19] S. Janson, T. Łuczak, A. Ruciński: Random Graphs. Wiley \& Sons, New York (2000).

[20] S. Jukna: Extremal Combinatorics with Applications in Computer Science. Springer, Heidelberg (2001).

[21] Ph. G. Kolaitis, M. Y. Vardi: Conjunctive-query containment and constraint satisfaction. In: Proc. of PODS'98 205-213.

[22] D. Král': Locally Satisfiable Formulas. In: Proc. of SODA'04 323-332.

[23] D. Král', O. Pangrác: An Asymptotically Optimal Linear-Time Algorithm for Locally Consistent Constraint Satisfaction Problems, Theoret. Comput. Sci. 348 (2005), 187-206. A preliminary version appeared: Proc. 30st International Symposium Mathematical Foundations of COmputer Science (MFCS), LNCS Vol. 3618, Springer-Verlag Berlin (2005), 603-614.

[24] B. Larose, C. Loten, C. Tardif: A characterisation of first-order constraint satisfaction problems, Logical Methods Comput. Sci. 3 (2007), 22pp.

[25] K. Lieberherr, E. Specker: Complexity of Partial Satisfaction. J. of the ACM, 28(2) (1981) 411-422.

[26] K. Lieberherr, E. Specker: Complexity of Partial Satisfaction II. Technical Report 293, Dept. of EECS, Princeton University (1982).

[27] R. Motwani, P. Raghavan: Randomized Algorithms. Cambridge University Press, 1995.

[28] L. Trevisan: On Local versus Global Satisfiability, SIAM J. Disc. Math. 17 (2004), 541-547.

[29] Z. Usiskin: Max-min Probabilities in the Voting Paradox. Ann. Math. Stat. 35 (1963) 857-862.

[30] G. J. Woeginger: Exact Algorithms for NP-hard Problems: A Survey. In: Proc. 5th International Workshop Combinatorial Optimization-Eureka, You Shrink. LNCS Vol. 2570. Springer-Verlag Berlin (2003) 185-207.

[31] M. Yannakakis: On the Approximation of Maximum Satisfiability. J. Algorithms 17 (1994) 475-502. A preliminary version appeared in Proc. 3rd Annual ACM-SIAM Symposium on Discrete Algorithms, SODA (1992) 1-9. 